\title{
Nonlinear waves of polarization in two-component Bose-Einstein condensates
}

\author{
A. M. Kamchatnov, ${ }^{1}$ Y. V. Kartashov, ${ }^{1}$ P.-É. Larré, ${ }^{2}$ and N. Pavloff ${ }^{2}$ \\ ${ }^{1}$ Institute of Spectroscopy, Russian Academy of Sciences, Troitsk, Moscow, 142190, Russia \\ ${ }^{2}$ Univ. Paris Sud, CNRS, Laboratoire de Physique Théorique et Modèles Statistiques, UMR8626, F-91405 Orsay, France
}

\begin{abstract}
Waves with different symmetries exist in two-component Bose-Einstein condensates (BECs) whose dynamics is described by a system of coupled Gross-Pitaevskii (GP) equations. A first type of waves corresponds to excitations for which the motion of both components is locally in phase. In the second type of waves the two components have a counter-phase local motion. In the case of different values of inter- and intra-component interaction constants, the long wave-length behavior of these two modes corresponds to two types of sound with different velocities. In the limit of weak nonlinearity and small dispersion the first mode is described by the well-known Korteweg-de Vries (KdV) equation. We show that in the same limit the second mode can be described by the Gardner (modified KdV) equation, if the intra-component interaction constants have close enough values. This leads to a rich phenomenology of nonlinear excitations (solitons, kinks, algebraic solitons, breathers) which does not exist in the KdV description.
\end{abstract}

PACS numbers: 47.37.+q,03.75.Mn,71.36.+c

\section{INTRODUCTION}

Two-component Gross-Pitaevskii (GP) equations describe the evolution of nonlinear excitations in various physical systems. Apparently, it first appeared in nonlinear optics as a "vector nonlinear Schrödinger equation" describing self-interaction of electromagnetic waves with account of their polarization (see, e.g., [1 3 ). In the case of equal nonlinearity constants, this system is completely integrable by the inverse scattering transform method 1 and many particular solutions have been found (see, e.g., [4] and references therein). Taking birefringence effects into account 5 leads to even richer dynamics, as confirmed by experiments on propagation of light pulses in fibers [6. The recent surge of interest in vector solitons was caused by the realization of spinor atomic BECs (see the reviews [7, 8] and references therein) as well as microcavity polariton condensates 9 .

From the physical point of view, a specific feature of two-component condensates is the existence of two types of elementary excitations. In one mode both condensates move locally in phase. In the case of a small amplitude potential flow this corresponds to usual sound waves consisting in density oscillations. In another "polarization" mode the two components move in counter-phase in such a way that, in some situations, the total density remains constant in spite of the excitation of relative motion of the condensate components. Correspondingly, these two modes can have different values of the sound velocities and, due to different symmetries, their excitation demand different methods [10, 11]. In some sense, this is analogous to the situation observed for the first and second sound in superfluid HeII: the second sound which corresponds to a temperature (and entropy) wave cannot be excited by oscillations of the container wall, contrarily to the usual density waves associated with the first sound; see, e.g., [12].

In the present paper, we study the weakly nonlinear evolution of these two modes in presence of small disper- sive effects. Whereas in this limit the density waves are described by the standard KdV equation which accounts for quadratic nonlinearities in the wave amplitude, the polarization mode is much more peculiar. We show that there are situations where one has to take into account third order nonlinearities in the wave amplitude and that the corresponding nonlinear polarization wave is then described by the Gardner equation. As is well known, this is a quite generic equation which arises when the coefficient in the quadratic nonlinearity term is small and when the wave amplitude has the same order of magnitude as this coefficient. In particular, the Gardner equation (and also the modified KdV equation which shares strong similarities with it) have been derived for interface waves in stratified fluid dynamics [13, 14, lattice dynamics described by the discrete nonlinear Schrödinger equation [15, and quantum dynamics of condensates in optical lattices [16]. The Gardner equation has a wide spectrum of different nonlinear excitations [17] which can be generated by the flow of fluid past an obstacle [18. We expect that the phenomenology associated to this rich dynamics can be observed in experiments with flows of BECs.

\section{MAIN EQUATIONS AND LINEAR WAVES}

We consider a two-component condensate confined in a one-dimensional structure (e.g., a cigar-shaped trap for atomic condensates or a quantum wire embedded into an elongated cavity for polariton condensate case) in which the BEC is described by a one-dimensional (1D) twocomponent order parameter $\left(\psi_{+}(x, t), \psi_{-}(x, t)\right)$ whose dynamics is modeled by a set of coupled Gross-Pitaevskii equations

$$
i \partial_{t} \psi_{ \pm}+\frac{1}{2} \partial_{x x}^{2} \psi_{ \pm}-\left[\left(\alpha_{1} \pm \delta\right)\left|\psi_{ \pm}\right|^{2}+\alpha_{2}\left|\psi_{\mp}\right|^{2}\right] \psi_{ \pm}=0
$$

written here in a standard non-dimensional form. The parameter $\delta$ measures the difference between the intra- 
species nonlinear interaction constants: $\alpha_{1}+\delta$ corresponds to the interaction among $\psi_{+}$particles and $\alpha_{1}-\delta$ to the one among $\psi_{-}$particles. It is supposed that the two species are labeled in such a way that $\delta>0 . \alpha_{2}$ is the inter-species interaction constant. In the case of atomic condensates, the two component order parameter may describe a two-species BEC such as realized by considering for instance ${ }^{87} \mathrm{Rb}$ in two hyperfine states [19, or a mixture of two elements 20, or different isotopes of the same atom 21. In the case of a polariton condensate, the components $\psi_{+}$and $\psi_{-}$account for the pseudo-spin of the polariton which consists in a resonant admixture of excitons and photons with spin projection \pm 1 .

It is convenient to introduce spinor variables $[22]$

$$
\left(\begin{array}{c}
\psi_{+} \\
\psi_{-}
\end{array}\right)=\sqrt{\rho} e^{i \Phi / 2} \chi=\sqrt{\rho} e^{i \Phi / 2}\left(\begin{array}{c}
\cos \frac{\theta}{2} e^{-i \phi / 2} \\
\sin \frac{\theta}{2} e^{i \phi / 2}
\end{array}\right) .
$$

Here $\rho(x, t)=\left|\psi_{+}\right|^{2}+\left|\psi_{-}\right|^{2}$ denotes the total density of the condensate and $\Phi(x, t)$ has the meaning of the velocity potential of its in-phase motion; the angle $\theta(x, t)$ is the variable describing the relative density of the two components $\left(\cos \theta=\left(\left|\psi_{+}\right|^{2}-\left|\psi_{-}\right|^{2}\right) / \rho\right)$ and $\phi(x, t)$ is the potential of their relative (counter-phase) motion. Accordingly, the densities of the components of the condensate are given by

$$
\rho_{+}(x, t)=\rho \cos ^{2}(\theta / 2), \quad \rho_{-}(x, t)=\rho \sin ^{2}(\theta / 2),
$$

their phases are defined as

$$
\varphi_{+}(x, t)=\frac{1}{2}(\Phi-\phi), \quad \varphi_{-}(x, t)=\frac{1}{2}(\Phi+\phi),
$$

and the corresponding velocities of each components are

$$
v_{+}(x, t)=\nabla \varphi_{+}, \quad v_{-}(x, t)=\nabla \varphi_{-} .
$$

It is also convenient to introduce a unit vector $\mathbf{S}$ representing the spinor $\chi$ :

$$
\mathbf{S}(x, t)=\chi^{\dagger} \boldsymbol{\sigma} \chi=\left(\begin{array}{c}
\sin \theta \cos \phi \\
\sin \theta \sin \phi \\
\cos \theta
\end{array}\right),
$$

where $\boldsymbol{\sigma}=\left(\sigma_{x}, \sigma_{y}, \sigma_{z}\right)^{T}$ is a vector of Pauli matrices. The vector $\mathbf{S}$ can be called the polarization vector of the two-component condensate.

Substitution of Eq. (2) into Eq. (1) yields the system

$$
\begin{aligned}
\rho_{t} & +\frac{1}{2}[\rho(U-v \cos \theta)]_{x}=0, \\
\Phi_{t} & -\frac{\cot \theta}{2 \rho}\left(\rho \theta_{x}\right)_{x}+\frac{\rho_{x}^{2}}{4 \rho^{2}}-\frac{\rho_{x x}}{2 \rho}+\frac{1}{4}\left(\Phi_{x}^{2}+\theta_{x}^{2}+\phi_{x}^{2}\right) \\
& +\rho\left(\alpha_{1}+\alpha_{2}+\delta \cos \theta\right)=0, \\
\rho \theta_{t} & +\frac{1}{2}\left[(\rho v \sin \theta)_{x}+\rho U \theta_{x}\right]=0, \\
\phi_{t} & -\frac{1}{2 \rho \sin \theta}\left(\rho \theta_{x}\right)_{x}+\frac{1}{2} U v-\rho\left[\delta+\left(\alpha_{1}-\alpha_{2}\right) \cos \theta\right]=0,
\end{aligned}
$$

where $U=\Phi_{x}$ and $v=\phi_{x}$ are the mean and the relative velocities: hence the velocities of the two components are equal to $\left.v_{ \pm}=(U \pm v) / 2\right)$. In what follows, we shall consider solitons and other nonlinear excitations corresponding to small deviations from a uniform quiescent condensate and, to simplify the treatment, we shall suppose that the two components in a non-excited condensate have equal densities. This can be realized by two choices $\theta \rightarrow \frac{\pi}{2}$ or $\theta \rightarrow \frac{3 \pi}{2}$ for $|x| \rightarrow \infty$. To be definite, we shall consider the case $\theta \rightarrow \frac{\pi}{2}$ since another choice leads to similar results when only signs of some parameters change. The other parameters of the wave must satisfy the boundary conditions

$$
\rho \rightarrow \rho_{0}, \quad U \rightarrow 0, \quad v \rightarrow 0 \quad \text { for } \quad|x| \rightarrow \infty .
$$

In the uniform state, the dependence of the phases on time is described by the factors $\psi_{ \pm} \propto \exp \left(-i \mu_{ \pm} t\right)$, where the chemical potentials $\mu_{ \pm}$are given by

$$
\begin{aligned}
& \mu_{+}=\frac{1}{8}(U-v)^{2}+\rho_{0}\left[\left(\alpha_{1}-\alpha_{2}+\delta\right) \cos ^{2}\left(\theta_{0} / 2\right)+\alpha_{2}\right] \\
& \mu_{-}=\frac{1}{8}(U+v)^{2}+\rho_{0}\left[\left(\alpha_{1}-\alpha_{2}-\delta\right) \sin ^{2}\left(\theta_{0} / 2\right)+\alpha_{2}\right]
\end{aligned}
$$

(for future convenience we keep here the general notation for $\theta_{0}$ ).

First, we shall consider linear waves propagating along uniform background. Linearization of the system (7) with respect to small variables $\rho^{\prime}=\rho-\rho_{0}, \theta^{\prime}=\theta-\pi / 2$, $U$ and $v$ yields

$$
\begin{aligned}
& \rho_{t}^{\prime}+\frac{1}{2} \rho_{0} U_{x}=0 \\
& U_{t}+\left(\alpha_{1}+\alpha_{2}\right) \rho_{x}^{\prime}-\frac{1}{2 \rho_{0}} \rho_{x x x}^{\prime}-\delta \rho_{0} \theta_{x}^{\prime}=0 \\
& \theta_{t}+\frac{1}{2} v_{x}=0 \\
& v_{t}+\rho_{0}\left(\alpha_{1}-\alpha_{2}\right) \theta_{x}^{\prime}-\frac{1}{2} \theta_{x x x}^{\prime}-\delta \rho_{x}^{\prime}=0 .
\end{aligned}
$$

One can notice that for $\delta=0$ the system 10 splits into two pairs of independent equations: the first pair describes the density waves with oscillations of $\rho$ and $U$ and the second pair describes the polarization waves with oscillations of relative density measured by $\theta$ and relative velocity $v$. For $\delta \neq 0$ these two modes are mixed but for convenience we will call them "density" and "polarization" waves if they transform into these waves in the limit $\delta \rightarrow 0$. If we look for the solution of the system (10) in the form of plane waves with all variables proportional to $\exp [i(k x-\omega t)]$, then we readily get the dispersion laws

$$
\begin{aligned}
& \omega_{d}^{2}(k)=\frac{1}{2} \rho_{0}\left(\alpha_{1}+\sqrt{\alpha_{2}^{2}+\delta^{2}}\right) k^{2}+\frac{1}{4} k^{4}, \\
& \omega_{p}^{2}(k)=\frac{1}{2} \rho_{0}\left(\alpha_{1}-\sqrt{\alpha_{2}^{2}+\delta^{2}}\right) k^{2}+\frac{1}{4} k^{4},
\end{aligned}
$$

for the density and polarization modes, correspondingly. In the long wavelength limit $k \rightarrow 0$ we obtain the expressions for the density and polarization sound velocities 


$$
\begin{aligned}
& c_{d, p}^{2}=\lim _{k \rightarrow 0}\left(\omega_{d, p}^{2}(k) / k^{2}\right) \\
& c_{d}^{2}=\frac{1}{2} \rho_{0}\left(\alpha_{1}+\sqrt{\alpha_{2}^{2}+\delta^{2}}\right), \\
& c_{p}^{2}=\frac{1}{2} \rho_{0}\left(\alpha_{1}-\sqrt{\alpha_{2}^{2}+\delta^{2}}\right) .
\end{aligned}
$$

In the case where $\delta \neq 0$, the degree of mixture between density and polarization waves can be evaluated by studying the dynamic structure factor $S(k, \omega)$ of the system. At zero temperature $S(k, \omega)=-\frac{1}{\pi} \Theta(\omega) \operatorname{Im} \chi(k, \omega)$ where $\Theta$ is the Heaviside step function and $\chi(k, \omega)$ is the density response function (see, e.g., Refs. 23] and 24]). $\chi(k, \omega)$ characterizes how the density of the system responds to a weak external scalar potential with wave vector $k$ and frequency $\omega$. In presence of such a perturbation, using a trivial modification of Eqs. 10 accounting for the effect of the external potential, one obtains

$$
\chi(k, \omega)=\frac{Z_{d}(k)}{\left(\omega+i 0^{+}\right)^{2}-\omega_{d}^{2}(k)}+\frac{Z_{p}(k)}{\left(\omega+i 0^{+}\right)^{2}-\omega_{p}^{2}(k)},
$$

with

$$
\begin{aligned}
& Z_{d}(k)=\frac{\rho_{0} k^{2}}{2}\left(1+\frac{\alpha_{2}}{\sqrt{\alpha_{2}^{2}+\delta^{2}}}\right) \text { and } \\
& Z_{p}(k)=\frac{\rho_{0} k^{2}}{2}\left(1-\frac{\alpha_{2}}{\sqrt{\alpha_{2}^{2}+\delta^{2}}}\right) .
\end{aligned}
$$

This yields

$S(k, \omega)=\frac{Z_{d}(k)}{2 \omega_{d}(k)} \delta\left(\omega-\omega_{d}(k)\right)+\frac{Z_{p}(k)}{2 \omega_{p}(k)} \delta\left(\omega-\omega_{p}(k)\right)$.

One has $\int_{\mathbb{R}} \omega S(k, \omega) d \omega=\frac{1}{2}\left(Z_{d}(k)+Z_{p}(k)\right)=\frac{1}{2} \rho_{0} k^{2}$ in agreement with the $f$-sum rule 23,24 . In the case where $\delta=0, Z_{p}(k)$ vanishes and the sum rule is exhausted by the peak at $\omega_{d}(k)$ : as stated above, this means that when $\delta=0$ the density fluctuations are completely described by the branch with dispersion $\omega_{d}(k)$. A further verification is that, in this case, the Feynman relation holds: $\omega_{d}(k)=k^{2} /\left(2 S_{k}\right)$ where $S_{k}=\int_{\mathbb{R}} S(k, \omega) d \omega[25$. When $\delta \neq 0$ the relative contribution to the density fluctuation of each branch can be evaluated by computing in which proportion the two peaks in (15) contribute to $S_{k}$. The ratio of these two contributions to $S_{k}$ is easily evaluated in the low and large $k$ limits. Provided one does not go in the Manakov regime described below, one sees in each of these two limiting cases that, for small $\delta$, the contribution to $S_{k}$ of the mode with dispersion $\omega_{p}(k)$ is lower by a factor of order $\left(\delta / \alpha_{2}\right)^{2}$ than the contribution of the mode with dispersion $\omega_{d}(k)$. Hence in the case of interest in the present work, where $\delta$ is small compared to $\alpha_{2}$, one can legitimately denote the branch with dispersion $\omega_{d}(k)$ the density modulation branch, and the one with dispersion $\omega_{p}(k)$ the polarization modulation branch.

In all the present work we suppose that

$$
\alpha_{1}^{2}>\alpha_{2}^{2}+\delta^{2},
$$

which is the condition of modulation stability of the polarization mode (see, e.g., Ref. [26]). Expressions for dispersion laws of linear waves propagating along binary condensates with non-equal background densities of two components were found in [27, 28].

As we can see from Eq. (12), in the Manakov regime where all nonlinearity constants are equal (i.e., $\delta=0$ and $\alpha_{1}=\alpha_{2}$ ) the polarization sound velocity vanishes. In this case the linear dispersion relation $\omega_{p} \approx c_{p} k$ can no longer be considered as correctly describing the dispersion relation in the long wavelength limit and the dispersive effects cannot be considered as small even when $k \rightarrow 0$. In the opposite configuration where the difference $\alpha_{1}-\left(\alpha_{2}^{2}+\delta^{2}\right)^{1 / 2}$ is large enough, then the regime of linear dispersion becomes of great importance when the characteristic value of the wave-vector $k$ satisfies the condition

$$
k^{2} \ll c_{d, p}^{2} .
$$

In this case, for linear waves propagating along the positive- $x$ direction, the dispersion laws 11 can be approximated by

$$
\omega_{d, p}(k) \cong c_{d, p} k+\frac{1}{8 c_{d, p}} k^{3} .
$$

Correspondingly, the wave amplitude, say, $\rho^{\prime}(x, t)$, satisfies the linear equation

$$
\rho_{t}^{\prime}+c_{d, p} \rho_{x}^{\prime}-\frac{1}{8 c_{d, p}} \rho_{x x x}^{\prime}=0
$$

where the last term describes a small dispersive correction to the propagation of pulses with constant sound speeds.

If the amplitude $\rho^{\prime}$ is small but finite and such that the last term in Eq. 190 has the same order of magnitude as the leading nonlinear correction (typically, $\left.\sim\left(\rho^{\prime}\right)^{2}\right)$, then nonlinear effects cannot be omitted for correctly describing the propagation of the pulse. This issue is addressed in the next section.

\section{EVOLUTION EQUATIONS FOR WEAKLY NONLINEAR WAVES IN A TWO-COMPONENT BEC}

\section{A. Nonlinear density waves}

We now take into account nonlinear effects in the propagation of a density pulse with an accuracy up to second order in the field variables $\rho^{\prime}, U, \theta^{\prime}, v$. To simplify the presentation, we omit the dispersive effects in a first 
stage. Then the system (7) reduces to

$$
\begin{aligned}
\rho_{t}^{\prime} & +\frac{1}{2} \rho_{0} U_{x}+\frac{1}{2} \rho_{0}\left(\theta^{\prime} v\right)_{x}+\frac{1}{2}\left(\rho^{\prime} U\right)_{x}=0, \\
U_{t} & +\left(\alpha_{1}+\alpha_{2}\right) \rho_{x}^{\prime}+\frac{1}{2}\left(U U_{x}+v v_{x}\right) \\
& -\delta \rho_{0} \theta_{x}^{\prime}-\delta\left(\rho^{\prime} \theta^{\prime}\right)_{x}=0, \\
\theta_{t}^{\prime} & +\frac{1}{2} v_{x}+\frac{1}{2 \rho_{0}} v \rho_{x}^{\prime}+\frac{1}{2} U \theta_{x}^{\prime}=0, \\
v_{t} & +\rho_{0}\left(\alpha_{1}-\alpha_{2}\right) \theta_{x}^{\prime}+\frac{1}{2}(U v)_{x} \\
& +\left(\alpha_{1}-\alpha_{2}\right)\left(\rho^{\prime} \theta^{\prime}\right)_{x}-\delta \rho_{x}^{\prime}=0 .
\end{aligned}
$$

Considering nonlinear density wave propagating in the positive- $x$ direction in the standard perturbation theory (see, e.g., 29]), one introduces the stretched variables

$$
\xi=\epsilon^{1 / 2}\left(x-c_{d} t\right), \quad \tau=\epsilon^{3 / 2} t
$$

and expand the fields variables $\rho^{\prime}, U, \theta^{\prime}, v$ in powers of $\epsilon$ :

$$
\begin{aligned}
& \rho^{\prime}=\epsilon \rho^{(1)}+\epsilon^{2} \rho^{(2)}+\ldots, \quad U=\epsilon U^{(1)}+\epsilon^{2} U^{(2)}+\ldots \\
& \theta^{\prime}=\epsilon \theta^{(1)}+\epsilon^{2} \theta^{(2)}+\ldots, \quad v=\epsilon v^{(1)}+\epsilon^{2} v^{(2)}+\ldots
\end{aligned}
$$

The corresponding expansion of Eqs. 20) yields, at leading order, the consistent system of equations

$$
\begin{aligned}
& -c_{d} \rho_{\xi}^{(1)}+\frac{1}{2} \rho_{0} U_{\xi}^{(1)}=0, \\
& -c_{d} U_{\xi}^{(1)}+\left(\alpha_{1}+\alpha_{2}\right) \rho_{\xi}^{(1)}-\delta \rho_{0} \theta_{\xi}^{(1)}=0, \\
& -c_{d} \theta_{\xi}^{(1)}+\frac{1}{2} v_{\xi}^{(1)}=0, \\
& -c_{d} v_{\xi}^{(1)}+\rho_{0}\left(\alpha_{1}-\alpha_{2}\right) \theta_{\xi}^{(1)}-\delta \rho_{\xi}^{(1)}=0 .
\end{aligned}
$$

The determinant of this linear system is zero, and all variables can thus be expressed it terms of one of them, $\rho^{(1)}$ for instance:

$$
\begin{aligned}
& U^{(1)}=\frac{2 c_{d}}{\rho_{0}} \rho^{(1)}, \quad \theta^{(1)}=\frac{\alpha_{2}-\sqrt{\alpha_{2}^{2}+\delta^{2}}}{\delta \rho_{0}} \rho^{(1)}, \\
& v^{(1)}=\frac{2 c_{d}}{\delta \rho_{0}}\left(\alpha_{2}-\sqrt{\alpha_{2}^{2}+\delta^{2}}\right) \rho^{(1)} .
\end{aligned}
$$

These are the relations actually realized in a linear density wave. At next order in $\epsilon$ we get

$$
\begin{aligned}
-c_{d} \rho_{\xi}^{(2)} & +\frac{1}{2} \rho_{0} U_{\xi}^{(2)} \\
& =-\rho_{\tau}^{(1)}-\frac{1}{2} \rho_{0}\left(\theta^{(1)} v^{(1)}\right)_{\xi}-\frac{1}{2}\left(\rho^{(1)} U^{(1)}\right)_{\xi}, \\
-c_{d} U_{\xi}^{(2)} & +\left(\alpha_{1}+\alpha_{2}\right) \rho_{\xi}^{(2)}-\delta \rho_{0} \theta_{\xi}^{(2)} \\
& =-U_{\tau}^{(1)}-\frac{1}{2}\left(U^{(1)} U_{\xi}^{(1)}+v^{(1)} v_{\xi}^{(1)}\right)+\delta\left(\rho^{(1)} \theta^{(1)}\right)_{\xi}, \\
-c_{d} \theta_{\xi}^{(2)} & +\frac{1}{2} v_{\xi}^{(2)} \\
& =-\theta_{\tau}^{(1)}-\frac{1}{2 \rho_{0}} v^{(1)} \rho_{\xi}^{(1)}-\frac{1}{2} U^{(1)} \theta_{\xi}^{(1)}, \\
-c_{d} v_{\xi}^{(2)} & +\rho_{0}\left(\alpha_{1}+\alpha_{2}\right) \theta_{\xi}^{(2)}-\delta \rho_{\xi}^{(2)} \\
& =-v_{\tau}^{(1)}-\frac{1}{2}\left(U^{(1)} v_{\xi}^{(1)}-\left(\alpha_{1}-\alpha_{2}\right)\left(\rho^{(1)} \theta^{(1)}\right)_{\xi} .\right.
\end{aligned}
$$

As already observed at order $\mathcal{O}(\epsilon)$ [compare with Eqs. [23)], the left-hand side of the system (25) has a vanishing determinant, hence the expressions in this side are linearly dependent. Therefore the expressions in the right-hand side must also be linearly dependent, with the same proportionality coefficients [which are the same as the ones already involved at order $\mathcal{O}(\epsilon)$ and explicitly written in Eqs. (24)]. This condition yields the evolution equation

$$
\rho_{\tau}^{(1)}+\frac{3\left(2 \sqrt{\alpha_{2}^{2}+\delta^{2}}-\alpha_{2}\right) c_{d}}{2 \rho_{0} \sqrt{\alpha_{2}^{2}+\delta^{2}}} \rho^{(1)} \rho_{\xi}^{(1)}=0 .
$$

We can now return to the previous coordinates $x$ and $t$ and take into account the dispersion effects by simply adding the dispersive term that was taken into account in Eq. 19p. This is legitimate because the only other possible quadratic and dispersive term $\sim \rho^{(1)} \rho_{\xi \xi}^{(1)}$ which could have be missed in our dispersionless approximation is forbidden since it does not have the same symmetry with respect to the transformation $x \rightarrow-x$ as the other terms. In other words, the inclusion of this term would result in different equations for left- and right-propagating waves. Thus, we arrive at the equation

$$
\rho_{t}^{\prime}+c_{d} \rho_{x}^{\prime}+\frac{3\left(2 \sqrt{\alpha_{2}^{2}+\delta^{2}}-\alpha_{2}\right) c_{d}}{2 \rho_{0} \sqrt{\alpha_{2}^{2}+\delta^{2}}} \rho^{\prime} \rho_{x}^{\prime}-\frac{1}{8 c_{d}} \rho_{x x x}^{\prime}=0,
$$

where $\rho^{\prime}(x, t)=\rho(x, t)-\rho_{0}$ and we work at a level of approximation where $\rho^{\prime}(x, t)=\epsilon \rho^{(1)}(x, t)$. If the solution of Eq. 27) is found, then the other variables can be obtained with the use of relations (24) which we rewrite for completeness with the final notations:

$$
\begin{aligned}
& U(x, t)=\frac{2 c_{d}}{\rho_{0}} \rho^{\prime}(x, t), \quad \theta^{\prime}(x, t)=\frac{\alpha_{2}-\sqrt{\alpha_{2}^{2}+\delta^{2}}}{\delta \rho_{0}} \rho^{\prime}(x, t), \\
& v(x, t)=\frac{2 c_{d}}{\delta \rho_{0}}\left(\alpha_{2}-\sqrt{\alpha_{2}^{2}+\delta^{2}}\right) \rho^{\prime}(x, t) .
\end{aligned}
$$

Equation (27) is the KdV equation for weakly nonlinear density waves. In the limit $\delta \rightarrow 0$ we get the equation

$\rho_{t}^{\prime}+c_{d} \rho_{x}^{\prime}+\frac{3 c_{d}}{2 \rho_{0}} \rho^{\prime} \rho_{x}^{\prime}-\frac{1}{8 c_{d}} \rho_{x x x}^{\prime}=0, \quad c_{d}=\sqrt{\frac{1}{2} \rho_{0}\left(\alpha_{1}+\alpha_{2}\right)}$

which in the case $\alpha_{2}=\alpha_{1}$ reduces to the $\mathrm{KdV}$ equation for shallow Manakov solitons.

\section{B. Nonlinear polarization waves: quadratic nonlinearity}

The equation of propagation of nonlinear polarization waves with account of quadratic nonlinearity can be obtained by the same method. We introduce the stretched variables

$$
\xi=\epsilon^{1 / 2}\left(x-c_{p} t\right), \quad \tau=\epsilon^{3 / 2} t
$$


and make use of the series expansions 22. At first order we obtain the system with $c_{d}$ replaced by $c_{p}$ which yields

$$
\begin{aligned}
& U^{(1)}=\frac{2 c_{p} \delta}{\alpha_{2}+\sqrt{\alpha_{2}^{2}+\delta^{2}}} \theta^{(1)}, \\
& \rho^{(1)}=\frac{\delta \rho_{0}}{\alpha_{2}+\sqrt{\alpha_{2}^{2}+\delta^{2}}} \theta^{(1)}, \\
& v^{(1)}=2 c_{p} \theta^{(1)} .
\end{aligned}
$$

These expressions are equivalent to Eqs. 24 — with $c_{d}$ replaced by $c_{p}$-but it is now more convenient to express all variables in terms of $\theta^{(1)}$. Tedious calculations at second order lead to the equation

$$
\theta_{\tau}^{(1)}+\frac{3 c_{p}\left(2 \delta^{2}+\alpha_{2}^{2}-\alpha_{2} \sqrt{\alpha_{2}^{2}+\delta^{2}}\right)}{2 \delta \sqrt{\alpha_{2}^{2}+\delta^{2}}} \theta^{(1)} \theta_{\xi}^{(1)}=0 .
$$

Returning to the coordinates $x$ and $t$ and taking the dispersive effects into account we arrive again at the $\mathrm{KdV}$ equation

$\theta_{t}^{\prime}+c_{p} \theta_{x}^{\prime}+\frac{3 c_{p}\left(2 \delta^{2}+\alpha_{2}^{2}-\alpha_{2} \sqrt{\alpha_{2}^{2}+\delta^{2}}\right)}{2 \delta \sqrt{\alpha_{2}^{2}+\delta^{2}}} \theta^{\prime} \theta_{x}^{\prime}-\frac{1}{8 c_{p}} \theta_{x x x}^{\prime}=0$

describing the dynamics of weakly dispersive and weakly nonlinear polarization waves. The other variables are expressed in terms of $\theta^{\prime}$ as follows:

$$
\begin{aligned}
& U(x, t)=\frac{2 c_{p} \delta}{\alpha_{2}+\sqrt{\alpha_{2}^{2}+\delta^{2}}} \theta^{\prime}(x, t), \\
& \rho^{\prime}(x, t)=\frac{\delta \rho_{0}}{\alpha_{2}+\sqrt{\alpha_{2}^{2}+\delta^{2}}} \theta^{\prime}(x, t), \\
& v(x, t)=2 c_{p} \theta^{\prime}(x, t) .
\end{aligned}
$$

Now, contrarily to the case of density waves exposed in Sec. III A the coefficient of the nonlinear term in (33) vanishes in the limit $\delta \rightarrow 0$ : in the limit $\delta \ll \alpha_{2}$ we get

$\theta_{t}^{\prime}+c_{p} \theta_{x}^{\prime}+\frac{9 c_{p} \delta}{4 \alpha_{2}} \theta^{\prime} \theta_{x}^{\prime}-\frac{1}{8 c_{p}} \theta_{x x x}^{\prime}=0, \quad c_{p}=\sqrt{\frac{\rho_{0}\left(\alpha_{1}-\alpha_{2}\right)}{2}}$.

This means that if $\theta^{\prime} \sim \delta \ll 1$, then the level of accuracy accepted here is not sufficient: the cubic nonlinearity terms $\sim\left(\theta^{\prime}\right)^{3}$ neglected in the present treatment have the same order of magnitude as the quadratic term in 35 . Thus, in the limit of small $\delta$ we have to consider the next order of approximation.

\section{Nonlinear polarization waves: cubic nonlinearity}

As advocated in Sec. IIIB, cubic nonlinearities becomes important when $\delta \sim \theta^{\prime}$ is small and their contribution can therefore be calculated from the system (7) with $\delta=0$. A series expansion up to the third order in the small variables $\rho^{\prime}, U, \theta^{\prime}, v$ and up to the first order in the derivatives of these quantities (we again postpone the inclusion of dispersive effect for simplifying the presentation) reads

$$
\begin{aligned}
\rho_{t}^{\prime} & +\frac{1}{2} \rho_{0} U_{x}+\frac{1}{2} \rho_{0}\left(\theta^{\prime} v\right)_{x}+\frac{1}{2}\left(\rho^{\prime} U\right)_{x}+\frac{1}{2}\left(\rho^{\prime} \theta^{\prime} v\right)_{x}=0 \\
U_{t} & +\left(\alpha_{1}+\alpha_{2}\right) \rho_{x}^{\prime}+\frac{1}{2}\left(U U_{x}+v v_{x}\right) \\
& +\frac{1}{2 \rho_{0}} \rho_{x}^{\prime}\left(\theta_{x}^{\prime}\right)^{2}-\frac{\left(\rho_{x}^{\prime}\right)^{3}}{2 \rho_{0}^{3}}=0 \\
\theta_{t}^{\prime} & +\frac{1}{2} v_{x}+\frac{1}{2 \rho_{0}} v \rho_{x}^{\prime}+\frac{1}{2} U \theta_{x}^{\prime} \\
& -\frac{1}{2 \rho_{0}^{2}} v \rho^{\prime} \rho_{x}^{\prime}-\frac{1}{2} v \theta^{\prime} \theta_{x}^{\prime}-\frac{1}{4}\left(\theta^{\prime}\right)^{2} v_{x}=0 \\
v_{t} & +\rho_{0}\left(\alpha_{1}-\alpha_{2}\right) \theta_{x}^{\prime}+\frac{1}{2}(U v)_{x}+\left(\alpha_{1}-\alpha_{2}\right)\left(\rho^{\prime} \theta^{\prime}\right)_{x} \\
& +\frac{1}{2 \rho_{0}^{2}}\left(\rho_{x}^{\prime}\right)^{2} \theta_{x}^{\prime}-\frac{\rho_{0}}{2}\left(\alpha_{1}-\alpha_{2}\right)\left(\theta^{\prime}\right)^{2} \theta_{x}^{\prime}=0
\end{aligned}
$$

We now introduce the stretched variables

$$
\xi=\epsilon^{1 / 2}\left(x-c_{p} t\right), \quad \tau=\epsilon^{5 / 2} t, \quad c_{p}=\sqrt{\frac{\rho_{0}\left(\alpha_{1}-\alpha_{2}\right)}{2}},
$$

and use the series expansions 22 up to the third order terms in $\epsilon$. At order $\mathcal{O}(\epsilon)$ we get

$$
\rho^{(1)}=0, \quad U^{(1)}=0, \quad v^{(1)}=2 c_{p} \theta^{(1)} .
$$

At next order we obtain the algebraic relations

$$
\begin{aligned}
& \rho^{(2)}=-\frac{3 c_{p}^{2}}{2 \alpha_{2}}\left(\theta^{(1)}\right)^{2}, \quad U^{(2)}=-\frac{c_{p}\left(3 \alpha_{1}+\alpha_{2}\right)}{2 \alpha_{2}}\left(\theta^{(1)}\right)^{2}, \\
& v^{(2)}=2 c_{p} \theta^{(2)}
\end{aligned}
$$

Finally, at order $\mathcal{O}\left(\epsilon^{3}\right)$ it is enough to consider the equations for $\theta^{\prime}$ and $v$ only:

$$
\begin{aligned}
-c_{p} \theta_{\xi}^{(3)} & +\frac{1}{2} v_{\xi}^{(3)}=-\theta_{\tau}^{(1)}-\frac{1}{2 \rho_{0}} \rho_{\xi}^{(2)} \\
& -\frac{1}{2} U^{(2)} \theta_{\xi}^{(1)}+\frac{1}{2} v^{(1)} \theta^{(1)} \theta_{\xi}^{(1)}+\frac{1}{4}\left(\theta^{(1)}\right)^{2} v_{\xi}^{(1)} \\
-c_{p} v_{\xi}^{(3)} & +\rho_{0}\left(\alpha_{1}-\alpha_{2}\right) \theta_{\xi}^{(3)}=-v_{\tau}^{(1)}-\frac{1}{2}\left(U^{(2)} v^{(1)}\right)_{\xi} \\
& -\left(\alpha_{1}-\alpha_{2}\right)\left(\theta^{(1)} \rho^{(2)}\right)_{\xi}+\frac{\rho_{0}}{2}\left(\alpha_{1}-\alpha_{2}\right)\left(\theta^{(1)}\right)^{2} \theta_{\xi}^{(1)} .
\end{aligned}
$$

Again the expressions in the left-hand side are linearly dependent and the compatibility condition for this system yields the evolution equation which, with account of Eqs. (38) and 39, can be written as

$$
\theta_{\tau}^{(1)}+\frac{3 c_{p}}{8}\left(1-9 \frac{\alpha_{1}}{\alpha_{2}}\right)\left(\theta^{(1)}\right)^{2} \theta_{\xi}^{(1)}=0
$$

Returning to the $(x, t)$-variables, and re-introducing dispersive effects according to the procedure exposed in 
Sec. III A, we arrive at the modified KdV equation

$$
\theta_{t}^{\prime}+c_{p} \theta_{x}^{\prime}-\frac{3 c_{p}}{8 \alpha_{2}}\left(9 \alpha_{1}-\alpha_{2}\right) \theta^{\prime 2} \theta_{x}^{\prime}-\frac{1}{8 c_{p}} \theta_{x x x}^{\prime}=0 .
$$

At last, if $\delta$ is small and $\theta^{\prime} \sim \delta$, we also have to take into account the quadratic nonlinearity of Eq. (35) and corrections of order $\mathcal{O}\left(\delta^{2}\right)$ to the velocity of the polarization sound:

$$
\begin{aligned}
\theta_{t}^{\prime} & +\left(c_{p}-\frac{\rho_{0} \delta^{2}}{8 c_{p} \alpha_{2}}\right) \theta_{x}^{\prime}+\frac{9 c_{p} \delta}{4 \alpha_{2}} \theta^{\prime} \theta_{x}^{\prime} \\
& -\frac{3 c_{p}}{8 \alpha_{2}}\left(9 \alpha_{1}-\alpha_{2}\right) \theta^{\prime 2} \theta_{x}^{\prime}-\frac{1}{8 c_{p}} \theta_{x x x}^{\prime}=0 .
\end{aligned}
$$

This is Gardner equation describing the evolution of nonlinear polarization pulses in a two-component condensate in the limit where the intra-species interaction constants are close. Once its solution is found, then the other variables are expressed in terms of $\theta^{\prime}$ by the formulas which follow from Eqs. (31), (38), and (39):

$$
\begin{aligned}
& \rho^{\prime}(x, t)=\frac{1}{2 \alpha_{2}}\left(\rho_{0} \delta \theta^{\prime}(x, t)-3 c_{p}^{2} \theta^{\prime 2}(x, t)\right), \\
& U(x, t)=\frac{c_{p}}{\alpha_{2}}\left(\delta \theta^{\prime}(x, t)-\frac{1}{2}\left(3 \alpha_{1}+\alpha_{2}\right) \theta^{\prime 2}(x, t)\right), \\
& v(x, t)=2 c_{p} \theta^{\prime}(x, t) .
\end{aligned}
$$

It is worth noticing that although in derivating the Gardner equation (43) we assumed the boundary condition $\theta^{\prime} \rightarrow 0$ as $|x| \rightarrow \infty$, this equation remains valid for the description of the evolution of waves with boundary conditions $\theta^{\prime} \rightarrow \theta_{1,2}$ as $x \rightarrow \pm \infty$ provided the values $\theta_{1,2}$ are small enough $\left(\left|\theta_{1,2}\right| \sim \delta \ll 1\right)$. As we shall see, this additional freedom makes it possible to obtain new types of solutions of the vector GP equation.

\section{WEAKLY NONLINEAR WAVES IN A TWO-COMPONENT BEC}

The system (1) admits a number of solutions with different properties depending on signs and values of $\alpha_{1}, \alpha_{2}, \delta$. The possible solutions can also depend on the background distributions of the condensate densities and velocities. Our boundary conditions $\rho_{ \pm} \rightarrow \rho_{0} / 2$ exclude such solutions as dark-bright solitons with vanishing at $|x| \rightarrow \infty$ density of the bright soliton component. These solutions have already been studied in the literature (see, e.g., Ref. 2, 3]) and have been observed in experiments [30 32. However, nonlinear coherent structures such as dark-dark solitons exist also in situations with nonvanishing at infinity background densities (see, e.g., 33]) and we shall provide here several new structures belonging to this class of solutions. To illustrate our approach by a simple example, we shall start with well-known darkdark density solitons described in the limit of shallow solitons by the KdV equation (27).

\section{A. Density KdV solitons}

Evolution of density waves is described by the $\mathrm{KdV}$ equation (27) and its well-known soliton solution is given in this case by the formula

$$
\begin{aligned}
\rho^{\prime}(x, t) & =-\frac{2 \rho_{0} \sqrt{\alpha_{2}^{2}+\delta^{2}}\left(c_{d}-V_{s}\right)}{c_{d}\left(2 \sqrt{\alpha_{2}^{2}+\delta^{2}}-\alpha_{2}\right)} \\
& \times \frac{1}{\cosh ^{2}\left[\sqrt{2 c_{d}\left(c_{d}-V_{s}\right)}\left(x-V_{s} t-x_{0}\right)\right]},
\end{aligned}
$$

where $c_{d}$ is defined by the first of Eqs. 121. From Eqs. (28) we can see that at $x \rightarrow \pm \infty$ the polarization vector $\mathbf{S}$ lies in the $\left(S_{x}, S_{y}\right)$-plane, it rotates in "southern hemisphere" of the $\mathbf{S}$-space with changing $x$, and its total rotation angle in the $\left(S_{x}, S_{y}\right)$-plane is equal to

$$
\Delta \phi=\frac{4 \sqrt{\alpha_{2}^{2}+\delta^{2}}\left(\sqrt{\alpha_{2}^{2}+\delta^{2}}-\alpha_{2}\right)}{\delta\left(2 \sqrt{\alpha_{2}^{2}+\delta^{2}}-\alpha_{2}\right)} \sqrt{1-\frac{V_{s}}{c_{d}}} .
$$

It goes to zero as $\Delta \phi \propto \sqrt{1-V_{s} / c_{d}}$ in the limit $V_{s} \rightarrow$ $c_{d}-0$ and as $\Delta \phi \propto \delta$ in the limit $\delta \rightarrow 0$.

If $\delta=0$, then the solution (45) reduces to the limit of shallow dark-dark soliton solution of the vector GP equation found in 28]. At last, if in addition $\alpha_{1}=\alpha_{2}$, then we reproduce the limit of shallow Manakov dark soliton [1]. It is important, that for $\delta=0$ the polarization variable $\theta$ remains constant and in this case $v \equiv 0$, so that, hence, the polarization vector $\mathbf{S}$ does not vary. We shall call this solution the density soliton even for $\delta \neq 0$ when the polarization vector rotates according to Eq. (46) since in the limit $\delta \rightarrow 0$ it transforms to a pure density mode.

\section{B. Polarization KdV solitons}

Now we turn to the polarization KdV solitons whose evolution is described by Eq. (33). The soliton solution is given by

$$
\begin{aligned}
\theta^{\prime}(x, t) & =-\frac{2 \delta \sqrt{\alpha_{2}^{2}+\delta^{2}}}{2 \delta^{2}+\alpha_{2}^{2}-\alpha_{2} \sqrt{\alpha_{2}^{2}+\delta^{2}}} \\
& \times \frac{1-V_{s} / c_{p}}{\cosh ^{2}\left[\sqrt{2 c_{p}\left(c_{p}-V_{s}\right)}\left(x-V_{s} t-x_{0}\right)\right]},
\end{aligned}
$$

where $c_{p}$ is defined by the second of equations 12 . Substitution of this expression into (34) allows one to find other parameters of solution. Again, if the phase $\phi$ is defined in such a way that $\phi=0$ at the center of the soliton, then the polarization vector rotates, as we go along the soliton solution, by the angle

$$
\Delta \phi=-\frac{2 \sqrt{2} \delta \sqrt{\alpha_{2}^{2}+\delta^{2}}}{\delta\left(2 \delta^{2}+\alpha_{2}^{2}-\alpha_{2} \sqrt{\alpha_{2}^{2}+\delta^{2}}\right)} \sqrt{1-\frac{V_{s}}{c_{d}}} .
$$

It goes to zero as $\Delta \phi \propto \sqrt{1-V_{s} / c_{d}}$ in the limit $V_{s} \rightarrow$ $c_{d}-0$, however, for $\delta \rightarrow 0$ this angle diverges as $\Delta \phi \propto \delta^{-1}$ 
what shows that the polarization mode ceases to exist in this limit. Such a behavior demonstrates drastic difference between density and polarization modes. The same remark refers to the amplitude of the polarization soliton (47). We can see that for small $\delta \ll\left|\alpha_{2}\right|$ the applicability condition of the small amplitude approximation $\left|\theta^{\prime}\right| \ll 1$ is satisfied provided that

$$
1-\frac{V_{s}}{c_{p}} \ll \frac{\delta}{\left|\alpha_{2}\right|}
$$

Even more heavy restriction follows from the condition that the soliton amplitude must be much smaller than the coefficient in the quadratic nonlinearity term so that we can neglect the cubic nonlinearity terms:

$$
1-\frac{V_{s}}{c_{p}} \ll\left(\frac{\delta}{\alpha_{2}}\right)^{2} .
$$

Thus, the applicability region of the $\mathrm{KdV}$ approximation is extremely small for $\delta \ll\left|\alpha_{2}\right|$ and we must take into account the cubic nonlinearity which corresponds to the Gardner equation 43.

\section{Polarization Gardner solitons}

The solution of the Gardner equation depends on signs and values of the nonlinear interaction constants $\alpha_{1}, \alpha_{2}, \delta$. In what follows we suppose that $\alpha_{1}>0$, $9 \alpha_{1}-\alpha_{2}>0, \alpha_{2}$ can be negative, and the condensate components are ordered in such a way that $\delta>0$.

At first we assume that $\alpha_{2}>0$. The soliton solution of the Gardner equation (43) is given by the formula (see, e.g., [17])

$$
\theta^{\prime}(x, t)=\frac{\theta_{1} \theta_{2}}{\theta_{1}-\left(\theta_{1}-\theta_{2}\right) \cosh ^{2}\left[\sqrt{2 c_{p} V}\left(x-V_{s} t\right)\right]},
$$

where

$$
c_{p}=\sqrt{\frac{1}{2} \rho_{0}\left(\alpha_{1}-\alpha_{2}\right)}, \quad V_{s}=c_{p}-\frac{\rho_{0} \delta^{2}}{8 c_{p} \alpha_{2}}-V,
$$

and

$$
\theta_{1,2}=\frac{6 \delta \pm 2 \sqrt{(3 \delta)^{2}+4 \alpha_{2}\left(9 \alpha_{1}-\alpha_{2}\right) V / c_{p}}}{9 \alpha_{1}-\alpha_{2}},
$$

where subscripts ' 1 ' and ' 2 ' correspond to the upper and lower signs, respectively, $V$ is a free parameter defining the velocity and other properties of the soliton solution (notice that $V$ measures the soliton velocity in the reference frame moving with the sound velocity equal to $\left.c_{p}-\rho_{0} \delta^{2} /\left(8 c_{p} \alpha_{2}\right)\right)$. Substitution of (51) into Eqs. 44 yields the density and polarization distributions of the polarization soliton.

Now the soliton's amplitude $\theta_{1}$ remains finite in the $\operatorname{limit} \delta \rightarrow 0$,

$$
\left.\theta_{1}\right|_{\delta=0}=4 \sqrt{\frac{\alpha_{2}}{9 \alpha_{1}-\alpha_{2}} \cdot \frac{V}{c_{p}}}
$$

and it is small for small enough $V$. The rotation angle of the polarization vector along the soliton solution is given by the expression

$$
\Delta \phi=8 \sqrt{\frac{2 \alpha_{2}}{9 \alpha_{1}-\alpha_{2}}} \arctan \left(\sqrt{-\frac{\theta_{1}}{\theta_{2}}}\right)
$$

and it is finite for all $\delta$ and $V$.
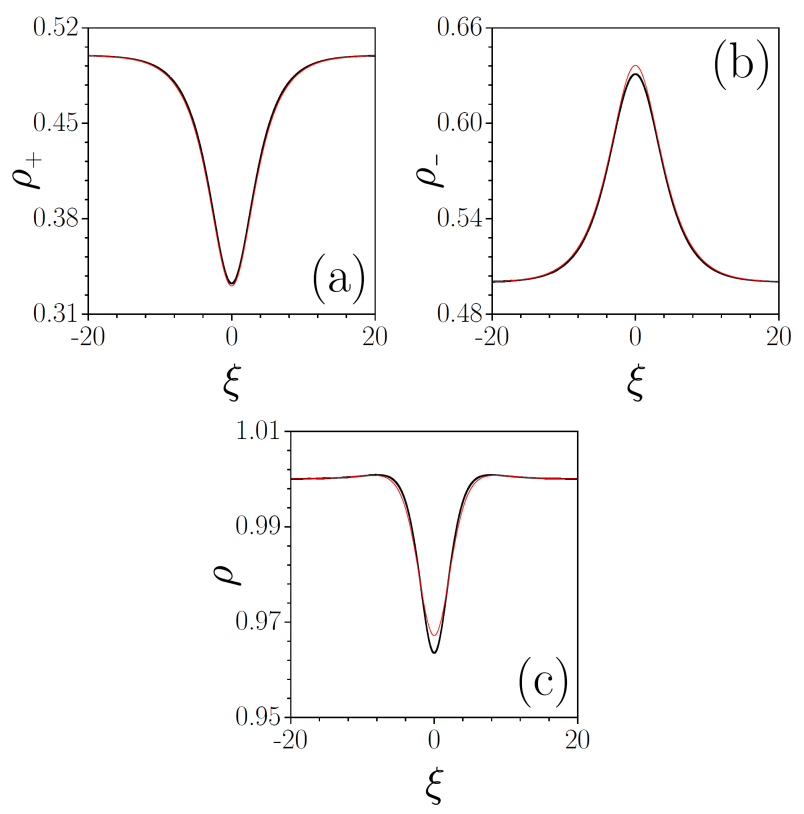

FIG. 1: (Color on line.) Dependence of (a) $\rho_{+}$, (b) $\rho_{-}$and the total density (c) $\rho$ on $\xi=x-V_{s} t$ for $\alpha_{1}=1.0, \alpha_{2}=0.6, \delta=$ 0.05 and velocity parameter $V=0.03$ (soliton velocity $V_{s}=$ 0.416). Analytical results in the Gardner approximation are shown by thick black lines and exact numerical results by red lines.

Densities of the two components of the soliton solution can be found from Eqs. (3). Their plots are illustrated in Fig. 1 together with exact numerical solutions of the vector GP equations for the same choice of parameters. Analogous plots for the flow velocities of two components are shown in Fig. 2.

In the center of a soliton and for $V \ll \rho_{0} \delta^{2} /\left(8 \alpha_{2} c_{p}\right)$, that is for soliton's velocity $V_{s}$ close to the linear polarization wave velocity $c_{p}$, the condensate's components densities are equal to

$$
\begin{array}{r}
\rho_{+}=\frac{\rho_{0}}{2}\left\{1-4 \sqrt{\frac{\alpha_{2}}{9 \alpha_{1}-\alpha_{2}}} \cdot \sqrt{1-\frac{V_{s}}{c_{p}}}\right. \\
\left.-\frac{12\left(\alpha_{1}-\alpha_{2}\right)}{9 \alpha_{1}-\alpha_{2}}\left(1-\frac{V}{c_{p}}\right)\right\}, \\
\rho_{-}=\frac{\rho_{0}}{2}\left\{1+4 \sqrt{\frac{\alpha_{2}}{9 \alpha_{1}-\alpha_{2}}} \cdot \sqrt{1-\frac{V_{s}}{c_{p}}}\right. \\
\left.-\frac{12\left(\alpha_{1}-\alpha_{2}\right)}{9 \alpha_{1}-\alpha_{2}}\left(1-\frac{V}{c_{p}}\right)\right\} .
\end{array}
$$



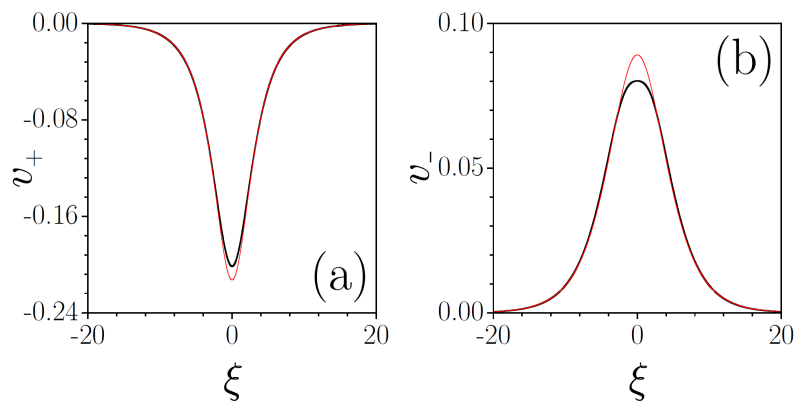

FIG. 2: (Color on line.) Dependence of (a) $v_{+}=(U-v) / 2$ and (b) $v_{-}=(u+v) / 2$ on $\xi=x-V_{s} t$ for $\alpha_{1}=1.0, \alpha_{2}=0.6, \delta=$ 0.05 and velocity parameter $V=0.03$ (soliton velocity $V_{s}=$ 0.416). Analytical results in the Gardner approximation are shown by thick black lines and exact numerical results by red lines.

These formulae agree with exact numerical solution of the vector GP equations (1) for $V_{s}$ close enough to $c_{p}$ (see Fig. 3).

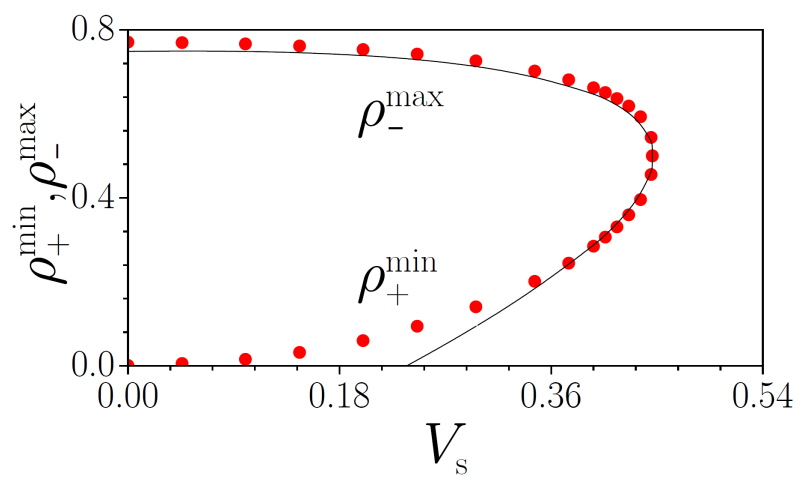

FIG. 3: (Color on line.) Dependence of two components densities at the center of soliton as functions of the soliton velocity $V_{s}$ for $\alpha_{1}=1.0, \alpha_{2}=0.6, \delta=0.05$. Analytical results in the Gardner approximation are shown by a solid line and exact numerical results by red dots.

If $\alpha_{2}<0$, the formula (51) for the soliton solution remains the same, but now in (53) subscripts ' 1 ' and ' 2 ' correspond to the lower and upper signs, respectively.

\section{Polarization algebraic solitons}

If $\alpha_{2}>0$, then there exists another type of soliton solutions of the Gardner equation - so called algebraic soliton (see, e.g., 17]). Such a solution is given by the expression

$$
\theta^{\prime}(x, t)=\theta_{2}+\frac{\theta_{1}-\theta_{2}}{1+\frac{c_{p}^{2}\left(9 \alpha_{1}-\alpha_{2}\right)\left(\theta_{1}-\theta_{2}\right)^{2}}{8 \alpha_{2}}\left(x-V_{s} t-x_{0}\right)},
$$

where $\theta_{2}$ is a free non-zero background density parameter which parameterizes the solution, and the other param- eters are defined by the equations

$$
\begin{gathered}
\theta_{1}=\frac{12 \delta}{9 \alpha_{1}-\alpha_{2}}-3 \theta_{2} \\
V_{s}=c_{p}-\frac{\delta^{2}}{8 c_{p} \alpha_{2}}+\frac{9 c_{p} \delta}{8 \alpha_{2}}-\frac{3 c_{p}\left(9 \alpha_{1}-\alpha_{2}\right)}{8 \alpha_{2}} \theta_{2}^{2} .
\end{gathered}
$$

In this case, for the background values of the angle $\theta_{2} \sim$ $\delta$, the soliton velocity departs from the sound velocity to a value $\sim \delta$, too, and, hence, the amplitude of the soliton is of the same order of magnitude. Thus, this is very small amplitude and very wide soliton.

\section{E. Solibore solutions}

Besides soliton solutions, similar in many respects to the KdV solitons, the Gardner equation has other types of solutions that are absent in the KdV approximation. Here we shall consider one such a solution, called "solibore" solution (see, e.g., [17, 34). In some applications the solutions of this kind are called "kinks" or "halfsolitons" 35, however in hydrodynamics they represent a degenerate case of a bore, i.e. of a solution that connects, analogously to a shock wave, two flows with different parameters. Exactly this meaning for the condensate flows has the solution that we are going to study here.

The solibore solution exists only for the case $\alpha_{1}>0$, $\alpha_{2}<0$, if the background flow is modulationally stable. In this subsection we shall assume that these conditions are fulfilled. In terms of the solution of the Gardner equation (43) the solibore solution relates the flows with different values of the angle $\theta$ at left and right infinities $x \rightarrow \pm \infty$ :

$$
\theta^{\prime} \rightarrow \theta_{1} \quad \text { at } \quad x \rightarrow+\infty \text { and } \theta^{\prime} \rightarrow \theta_{2} \text { at } x \rightarrow-\infty \text {. }
$$

This replaces the boundary condition $\theta \rightarrow \pi / 2$ presumed earlier, but the Gardner equation is still applicable provided $\left|\theta_{1,2}-\pi / 2\right|$ are small enough. In the solibore solution the limiting values $\theta_{1,2}$ are related by the expression

$$
\theta_{1}+\theta_{2}=\frac{3 c_{p} \delta}{8\left|\alpha_{2}\right|}
$$

For definiteness we suppose that $\theta_{1}>\theta_{2}$ and $\theta^{\prime} \rightarrow \theta_{1}$ at $x \rightarrow+\infty$; then the solibore solution of the Gardner equation can be written as

$$
\theta^{\prime}(x, t)=\theta_{1}-\frac{\theta_{1}-\theta_{2}}{1+\exp \left[\sqrt{\frac{9 \alpha_{1}-\alpha_{2}}{2\left|\alpha_{2}\right|}}\left(\theta_{1}-\theta_{2}\right)\left(x-V_{s} t-x_{0}\right)\right]},
$$

where the solibore velocity equals to

$$
\begin{aligned}
V_{s}=c_{p}-\frac{\rho_{0} \delta^{2}}{8 \alpha_{2} c_{p}} & +\frac{\left(9 \alpha_{1}-\alpha_{2}\right) c_{p}}{16 \alpha_{2}} \\
& \times\left[\left(\frac{6 \delta}{9 \alpha_{1}-\alpha_{2}}+\theta_{1}\right)^{2}-3 \theta_{1}^{2}\right] .
\end{aligned}
$$


This solution is parameterized by the value of the angle $\theta_{1}$ at the right infinity $x \rightarrow+\infty$; then $\theta_{2}$ is defined by Eq. (61). The other variables can be found by substitution of Eq. (62) into Eqs. (44). We have illustrated the solibore solution by plots in Figs. 4. As one can see, this solution represents a two-fluid flow which converts one component to another: downstream the solibore (on the left side) the $\rho_{+}$-component dominates and upstream the $\rho_{-}$-component dominates. In terms of the total density, the solibore solution looks as an asymmetric dark soliton moving with velocity $V_{s}$. It is important to notice that velocities of two components are different and do not vanish at $x \rightarrow \pm \infty$.
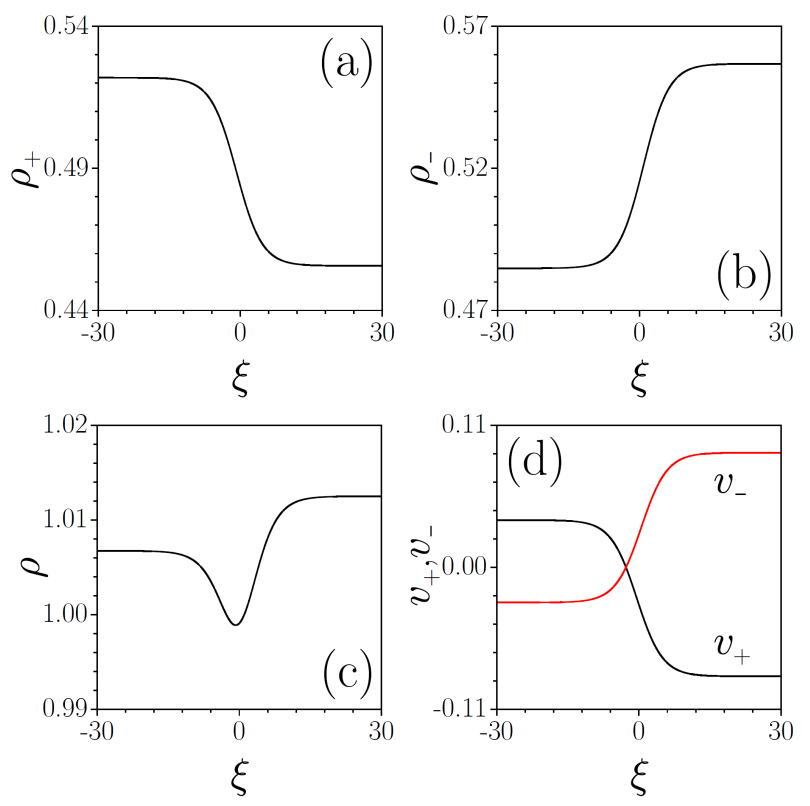

FIG. 4: (Color on line.) Dependence of $\rho_{+}(\mathrm{a}), \rho_{-}(\mathrm{b})$, the total density $\rho$ (c) and condensate's components velocities $v_{+}=(U-v) / 2, v_{-}=(u+v) / 2(\mathrm{~d})$ on $\xi=x-V_{s} t$ for $\alpha_{1}=1.0, \alpha_{2}=-0.6, \delta=0.05$ and $\theta_{1}=0.05$ (solibore velocity $\left.V_{s}=0.896\right)$. Exact numerical solution of the vector GP equations cannot be distinguished from the analytical results obtained in the Gardner approximation and therefore they are not shown here.

\section{F. Breather solution}

As the last example of new wave structures supported by the vector GP equation, we shall present the breather solution which appears as a consequence of the corresponding solution of the Gardner equation, found in [36. 37. In our notation it exists if $\alpha_{2}>0$ and can be written in the form

$$
\begin{aligned}
\theta^{\prime}(x, t) & =-\frac{2}{c_{p}} \sqrt{\frac{2 \alpha_{2}}{9 \alpha_{1}-\alpha_{2}}} \\
& \times \frac{\partial}{\partial x} \arctan \frac{\kappa \cosh p \cos \Theta_{b}-k \cos q \sinh \Phi_{b}}{\kappa \sinh p \sin \Theta_{b}+k \sin q \cosh \Phi_{b}}
\end{aligned}
$$

where $k$ and $\kappa$ are "wavenumbers" of an envelope and a carrier wave, correspondingly,

$$
\begin{aligned}
& k=\frac{3 \delta c_{p}}{\sqrt{2 \alpha_{2}\left(9 \alpha_{1}-\alpha_{2}\right)}} \frac{\sinh (2 p)}{\cos ^{2} q \cosh ^{2} p+\sin ^{2} q \sinh ^{2} p}, \\
& \kappa=\frac{3 \delta c_{p}}{\sqrt{2 \alpha_{2}\left(9 \alpha_{1}-\alpha_{2}\right)}} \frac{\sin (2 q)}{\cos ^{2} q \cosh ^{2} p+\sin ^{2} q \sinh ^{2} p},
\end{aligned}
$$

and velocities of the envelope and carrier wave are given by the expressions

$V_{b}=c_{p}-\frac{\rho_{0} \delta^{2}}{8 c_{p} \alpha_{2}}-\frac{3 \kappa^{2}-k^{2}}{8 c_{p}}, \quad V_{i}=c_{p}-\frac{\rho_{0} \delta^{2}}{8 c_{p} \alpha_{2}}-\frac{\kappa^{2}-3 k^{2}}{8 c_{p}}$,

while the phases are defined as

$$
\Theta_{b}=k\left(v-V_{b} t\right)+\Theta_{0}, \quad \Phi_{b}=\kappa\left(x-V_{i} t\right)+\Phi_{0} .
$$

Substitution of (64) into Eqs. (3) yields the densities of two components in the breather solution of vector GP equation in the Gardner approximation. This solution is illustrated in Fig. 5. It describes a non-stationary propagation of a nonlinear wave packet with envelope velocity $V_{b}$.
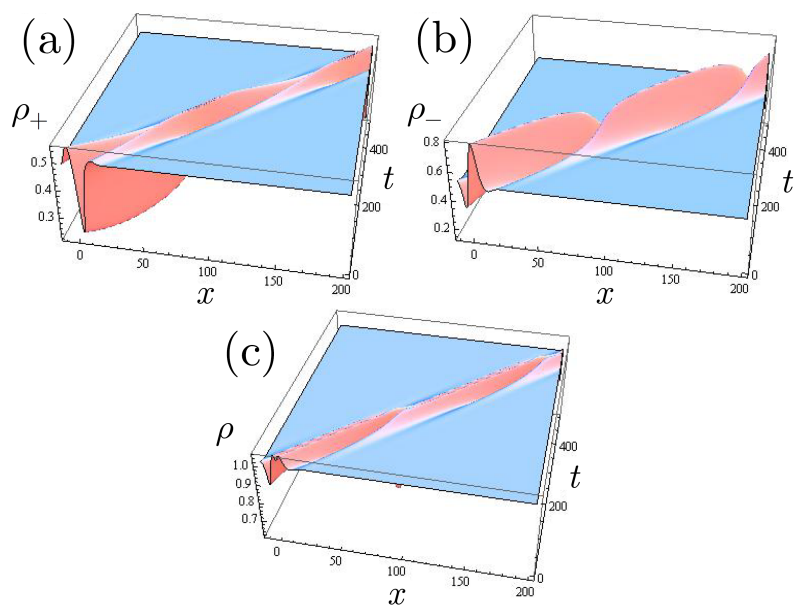

FIG. 5: (Color on line.) Plots of $\rho_{+}(\mathrm{a}), \rho_{-}(\mathrm{b})$, and the total density $\rho$ (c) as functions of $x$ and $t$ for $\alpha_{1}=1.0, \alpha_{2}=$ $0.6, \delta=0.4$.

\section{CONCLUSION}

In this paper, we have shown that for vector GP equation there exists a region of values of the nonlinearity 
constants for which this quite complicated system of equations can be reduced to a single nonlinear evolution equation - the so-called Gardner equation. This equation describes the evolution of the nonlinear polarization excitations, that is of such excitations which in the linear limit reduce to the polarization sound waves with constant total density of the condensate. Such an approach leads to several new types of nonlinear waves in the two-component condensates - algebraic solitons, solibores, breathers. Numerical solution of the vector GP equation with corresponding initial data demonstrates that these new types of nonlinear excitations propagate without change of their properties during evolution, that is they are stable with respect to decay to more elementary excitations. Although analytical description of the wave pattern exists at the moment in the small amplitude approximation only, similar large amplitude wave patterns can be described as exact numerical solutions of the vector GP equation. We expect that these new excitations can be generated in experiments by the flow of the two-component condensate past obstacles or by the phase and density engineering methods applied to the two-component condensate.

\section{Acknowledgments}

AMK thanks Laboratoire de Physique Théorique et Modèles Statistiques, Université Paris-Sud, Orsay, where this work was started, for kind hospitality. This work was supported by the French ANR under grant $n^{\circ}$ ANR11-IDEX-0003-02 (Inter-Labex grant QEAGE).
[1] S. V. Manakov, Zh. Eksp. Teor. Fiz. 65, 505 (1973) [Sov. Phys. JETP, 38, 248 (1974)].

[2] A. P. Sheppard and Y. S. Kivshar, Phys. Rev. E, 55, 4773 (1997).

[3] Y. S. Kivshar, G. Agraval, Optical Solitons: From Fibers to Photonic Crystals, (Academic Press, 2003).

[4] Q.-H. Park and H. J. Shin, Phys. Rev. E, 61, 3093 (2000).

[5] C. R. Menyuk, Optics Lett., 12, 614 (1987)

[6] S. T. Cundiff, B. C. Collings, N. N. Akhmediev, J. M. Soto-Crespo, K. Bergman, and W. H. Knox, Phys. Rev. Lett. 82, 3988 (1999).

[7] Y. Kawaguchi and M. Ueda, Phys. Rep., 520, 253 (2012).

[8] D. M. Stamper-Kurn and M. Ueda, Rev. Mod. Phys. 85, 1191 (2013).

[9] R. Hivet, H. Flayac, D. D. Solnyshkov, D. Tanese, T. Boulier, D. Andreoli, E. Giacobino, J. Bloch, A. Bramati, G. Malpuech, and A. Amo, Nature Phys., 8, 724 (2012).

[10] H. Flayac, H. Terças, D. D. Solnyshkov, and G. Malpuech, arXiv:1212.5894

[11] P.-É. Larré, N. Pavloff, and A. M. Kamchatnov, (to be published).

[12] L. D. Landau and E. M. Lifshitz, Fluid Mechanics, (Pergamon, 1959).

[13] T. Kakutani and N. Yamasaki, J. Phys. Soc. Japan., 45, 674 (1978).

[14] W. K. Melville and K. R. Helfrich, J. Fluid Mech., 178, 31 (1987).

[15] A. M. Kamchatnov, A. Spire, V. V. Konotop, J. Phys. A: Math. Gen. 37, 5547 (2004).

[16] E. Demler and A. Maltsev, Ann. Phys., 326, 1775 (2011).

[17] A. M. Kamchatnov, Y.-H. Kuo, T.-C. Lin, T.-L. Horng, S.-C. Gou, R. Clift, G. A. El, and R. H. J. Grimshaw, Phys. Rev. E 86, 036605 (2012).

[18] A. M. Kamchatnov, Y.-H. Kuo, T.-C. Lin, T.-L. Horng, S.-C. Gou, R. Clift, G. A. El, and R. H. J. Grimshaw, Transcritical flow of a stratified fluid over topography: analysis of the forced Gardner equation, arXiv:1305.3316 (2013).

[19] C. J. Myatt, E. A. Burt, R. W. Ghrist, E. A. Cornell, and C. E. Wieman, Phys. Rev. Lett. 78, 586 (1997).

[20] G. Modugno G., M. Modugno, F. Riboli, G. Roati G. and M. Inguscio, Phys. Rev. Lett. 89, 190404 (2002).

[21] S. B. Papp, J. M. Pino and C. E. Wieman, Phys. Rev. Lett. 101, 040402 (2008).

[22] K. Kasamatsu, M. Tsubota, and M. Ueda, Phys. Rev. A, 71, 043611 (2005).

[23] D. Pines and P. Nozières, The Theory of Quantum Liquids (Benjamin, New-York, 1966).

[24] L. Pitaevskii and S. Stringari, Bose-Einstein Condensation (Clarendon Press, Oxford, 2003).

[25] R. P. Feynman, Phys. Rev. 94, 262 (1954).

[26] C. J. Pethick and H. Smith, Bose-Einstein Condensation in Dilute Gases, (Cambridge University Press, Cambridge, 2002).

[27] D. V. Fil and S. I. Shevchenko, Phys. Rev. A 72, 013616 (2005).

[28] Yu. G. Gladush, A. M. Kamchatnov, Z. Shi, P. G. Kevrekidis, D. J. Frantzeskakis, B. A. Malomed, Phys. Rev. A 79, 033623 (2009).

[29] A. M. Kamchatnov, Nonlinear Periodic Waves and Their Modulations - An Introductory Course, (World Scientific, Singapore, 2000).

[30] B. P. Anderson, P. C. Haljan, C. A. Regal, D. L. Feder, L. A. Collins, C. W. Clark, and E. A. Cornell, Phys. Rev. Lett. 86, 2926 (2001).

[31] C. Becker, S. Stellmer, P. Soltan-Panahi, S. Dörscher, M. Baumert, E.-M. Richter, J. Kronjäger, K. Bongs, and K. Sengstock, Nature Physics 4, 496 (2008).

[32] C. Hamner, J. J. Chang, P. Engels, and M. A. Hoefer, Phys. Rev. Lett. 106, 065302 (2011).

[33] M. A. Hoefer, J. J. Chang, C. Hamner, and P. Engels, Phys. Rev. A 84, 041605(R) (2011).

[34] P. E. Holloway, E. Pelinovsky and T. Talipova, J. Geoph. Res., 104, 18-333 (1999).

[35] H. Flayac, D. D. Solnyshkov, and G. Malpuech, Phys. Rev. B 83, 193305 (2011).

[36] D. E. Pelinovsky, R. H. J. Grimshaw, Phys. Lett. A 229, 165 (1997).

[37] R. Grimshaw, D. Pelinovsky, E. Pelinovsky, T. Talipova, Physica D 159, 35 (2001). 